\title{
Parametrial tumour causing hydronephrosis and hydroureter due to IgG4 mediated retroperitoneal fibrosis; a laparoscopic resection
}

\author{
Dhammike Silva $^{1}$, Prabath Randombage ${ }^{2}$, Wedisha Gankanda ${ }^{2}$, Rukshani Edirisinghe ${ }^{2}$, Janani \\ Mathanamohan ${ }^{2}$, Chandu De Silva $^{3}$
}

\begin{abstract}
Retroperitoneal fibrosis (RPF) is a disease that develops following inflammation and fibrosis of the retroperitoneum and other abdominal organs, which manifests with various local and systemic symptoms. It has several entities, namely the idiopathic RPF (IRPF), secondary RPF and Immunoglobulin G4-related disease (IgG4-RD). The role of a gynaecologist in the management of retroperitoneal fibrosis is highlighted in this case of a parametrial tumour causing retroperitoneal fibrosis and hence leading to hydronephrosis and hydroureter as a consequence.
\end{abstract}

A 49-year-old woman presenting with a symptom cluster suggestive of acute pyelonephritis was found to have reduced eGFR. Elevated levels of inflammatory markers indicated an inflammatory nature of the disease, in addition to a normochromic anaemia that could be due to chronic inflammation itself. CECT Neck, Chest, Abdomen and Pelvis revealed mild hydronephrosis with a distal narrowing and a retroperitoneal confluent mass. Investigations led to suspect an invasive left ovarian malignancy or an endometriotic nodule with ureteric compression, which was later thought to be unlikely with a normal CA-125 level.

To obtain a better understanding, a cysto-ureteroscopy was performed where she was found to have an obstruction. Although stent placement is a reasonable temporary mode of symptom relief, this was proven unsuccessful. Surgical management was opted for considering the severity of her symptoms and the course of time it would take for medical management to take effect. In addition, laparoscopy would aid in the exclusion of other pelvic pathologies under direct vision and treat any if found concurrently.

Consequently, a laparoscopic hysterectomy, bilateral salpingo-oophorectomy, left sided ureterolysis with careful resection of the densely adhered tumour surrounding the left cysto-ureteric junction was performed, followed by retrograde ureteric stenting. Histology revealed retro-peritoneal fibrosis, and further immuno- histochemistry pointed towards a diagnosis of IgG4 related sclerosing disease. This was also supported by elevated levels of IgG, especially the IgG4 component. Disseminated RPF was subsequently excluded by CECT.

Following surgery, she was started on high dose corticosteroids and the stent was left in place for six weeks. She is being kept on a maintenance dose of low dose corticosteroids without disease relapse. Regular follow-up with inflammatory markers, serum IgG4 levels and imaging demonstrate adequate response to corticosteroid therapy.

Ceylon Medical Journal 2021; 66: 55-58

DOI: http://doi.org/10.4038/cmj.v66i1.9360

\section{Introduction}

Retroperitoneal fibrosis (RPF) is a rare disease characterized by the development of inflammation and fibrosis in the soft tissues of the retroperitoneum and other abdominal organs. There will be a series of clinical manifestations once the fibro-inflammatory tissue encases the abdominal aorta, iliac arteries and urinary tract [1].

RPF is generally divided into two types: idiopathic RPF (IRPF) and secondary RPF. Immunoglobulin G4related disease (IgG4-RD) is a new addition to the above and was officially named in 2010. The aetiology of this fibro-inflammatory disorder is characterized by elevated serum IgG4 concentration, infiltration of huge amounts of lymphocytes and IgG4+ plasma cells, while fibrosis in lesions remain unknown [2].

IgG4 mediated retroperitoneal fibrosis demonstrates a range of clinical manifestations such as lower back pain, abdominal pain or flank pain with urinary symptoms, which may mimic a renal colic and also exhibit systemic symptoms such as fever, malaise, nausea, vomiting, weight

${ }^{1}$ Department of Obstetrics and Gynaecology, Faculty of Medical Sciences, University of Sri Jayewardenepura, Sri Lanka, '2Department of Obstetrics and Gynaecology, Colombo South Teaching Hospital, Kalubowila, Sri Lanka, ${ }^{3}$ Department of Pathology, Faculty of Medicine, University of Colombo, Sri Lanka.

Correspondence: PR, e-mail: <prabathrandombage@gmail.com>. Received 22 October 2020 and revised version 19 February 2021 accepted 20 March 2021.

This is an open-access article distributed under the terms of the Creative Commons Attribution License, which permits unrestricted use, distribution, and reproduction in any medium, provided the original author and source are credited. 
loss, lower extremity oedema and less commonly claudication $[2,3]$.

There is a comprehensive diagnostic criteria for IgG4RD: (i) clinical examination showing characteristic diffuse/ localized swelling or masses in single or multiple organs; (ii) haematological examination showing elevated serum IgG4 concentrations (> $1350 \mathrm{mg} / \mathrm{L}$ ); and (iii) histopathologic examination showing marked lymphocyte and plasmocytic infiltration and fibrosis, as well as infiltration of IgG4+ plasma cells at a ratio of IgG4/ IgG cells $>40 \%$ and $>10$ IgG4+ plasma cells/high- power field (definite: $1+2+3$; probable: $1+3$; possible: $1+2$ ) [3].

This paper presents the very rare presentation of IgG4-related RPF of the parametrium resulting in hydronephrosis and hydroureter which ultimately resulted in laparoscopic resection.

\section{Case history}

A 49-year-old female presented with a brief history of fever with chills, left-sided flank pain and urinary symptoms, which resembled a clinical picture of acute pyelonephritis. On admission, her C-Reactive Protein (CRP) was $96.0 \mathrm{mg} / \mathrm{L}$ (0-5.0), and Erythrocyte Sedimentation Rate (ESR) was $73 \mathrm{~mm} / 1$ st hour (0-15). Elevated inflammatory markers were suggestive of an inflammatory nature of the disease. Estimated glomerular filtration rate (eGFR) was decreased $(60.42 \mathrm{ml} / \mathrm{min} / 1.73 \mathrm{~m} 2)$ with a normal serum creatinine level of 1.08. Full blood count revealed a normochromic anaemia with a Haemoglobin level of $9.2 \mathrm{mg} / \mathrm{dL}$, Mean Corpuscular Volume (MCV) of 81.8, Mean Corpuscular Haemoglobin (MCH) of 26.2, Mean Corpuscular Haemoglobin Concentration (MCHC) of 32.1 and Red Cell Distribution Width (RDW) of $17.9 \%$, possibly related to chronic inflammation. She has undergone a myomectomy by laparotomy in 2014 . There is no history of any chronic allergic conditions.

A Contrast Enhanced Computed Tomography (CECT) of the neck, chest, abdomen and pelvis revealed mild hydronephrosis of the left kidney with a narrowing of the distal ureter at S2 level caused by a retroperitoneal confluent mass. A negative polymerase chain reaction (PCR) for tuberculosis (TB) excluded the possibility of urogenital TB. Urine cytology for malignant cells was negative invoking a provisional diagnosis of an invasive left ovarian malignancy or an endometriotic nodule with ureteric compression. Both of the aforementioned conditions are expected to result in an elevated CA-125 level, which however, was normal in this patient, at $28 \mathrm{U} / \mathrm{mL}$.

Cysto-ureteroscopy was performed prior to laparoscopy and she was found to have an obstruction about 1-2 cm above the left cysto-ureteric junction where the stenting had failed. Laparoscopic hysterectomy, bilateral salpingo-oophorectomy, left sided ureterolysis with careful resection of the densely adhered tumour surrounding the left cysto-ureteric junction was performed. The segment of bladder with tumour involvement was resected as well and this necessitated retrograde ureteric stenting (Figure 1).

Histopathology was compatible with Retro Peritoneal Fibrosis (RPF) associated with a plasma cell infiltrate, arteritis and obliterative phlebitis that favour a diagnosis of IgG4 mediated sclerosing disease (Figure 2).

Following this, serum IgG4 levels were measured to support the diagnosis and revealed high values of IgG1 at $12.83 \mathrm{~g} / \mathrm{L}$ (3.2 - 10.2), IgG2 at $7.44 \mathrm{~g} / \mathrm{L}$ (1.2 - 6.6), IgG3 at 2.3 $\mathrm{g} / \mathrm{L}(0.2$ - 1.9) and IgG4 at $6.16 \mathrm{~g} / \mathrm{L}(<1.3)$ were found to be present.

Immunohistochemistry for IgG4 was performed which revealed elevated levels of IgG4 plasma cells over other IgG plasma cells. The proportion of CD138 positive plasma cells that are IgG4 positive were greater, which confirmed that the RPF is likely due to IgG4 related disease [4]. The histopathology was consistent with vasculitis and obliterative phlebitis in the absence of neoplastic cells scattered among the fibrous tissue which points towards the possibility of an idiopathic nature of the RPF. Furthermore, serum Amyloid A level was elevated with a negative ANA result.

The CECT of the rest of the body including neck, chest, abdomen and pelvis did not reveal any evidence of fibrosis in other sites which excluded disseminated RPF.

She was initially started on high dose corticosteroids; 60 milligrams of methylprednisolone daily for two months with the aim of suppressing the fibro-inflammatory reaction. The ureteric stent was kept for about six weeks and continuous follow-up care was provided. She had good clinical response, as demonstrated by gradually declining levels of inflammatory markers such as ESR and CRP performed every four weeks and is currently being managed on low dose steroid therapy without the need for additional corticosteroid-sparing agents to control the disease. A repeat CECT done three months later revealed a reduction in the degree of hydronephrosis, and is planned to undergo further imaging every three months until disease stability is achieved, following which six monthly imaging would deem adequate. Serum IgG4 levels were measured again after 6 weeks, and showed a decline from $6.16 \mathrm{~g} / \mathrm{L}$ to $3.2 \mathrm{~g} / \mathrm{L}$, which indicates good response to treatment.

The patient was counselled on natural disease progression, the benefits and adverse effects of the treatment being given and the importance of drug compliance in order to prevent complications. 


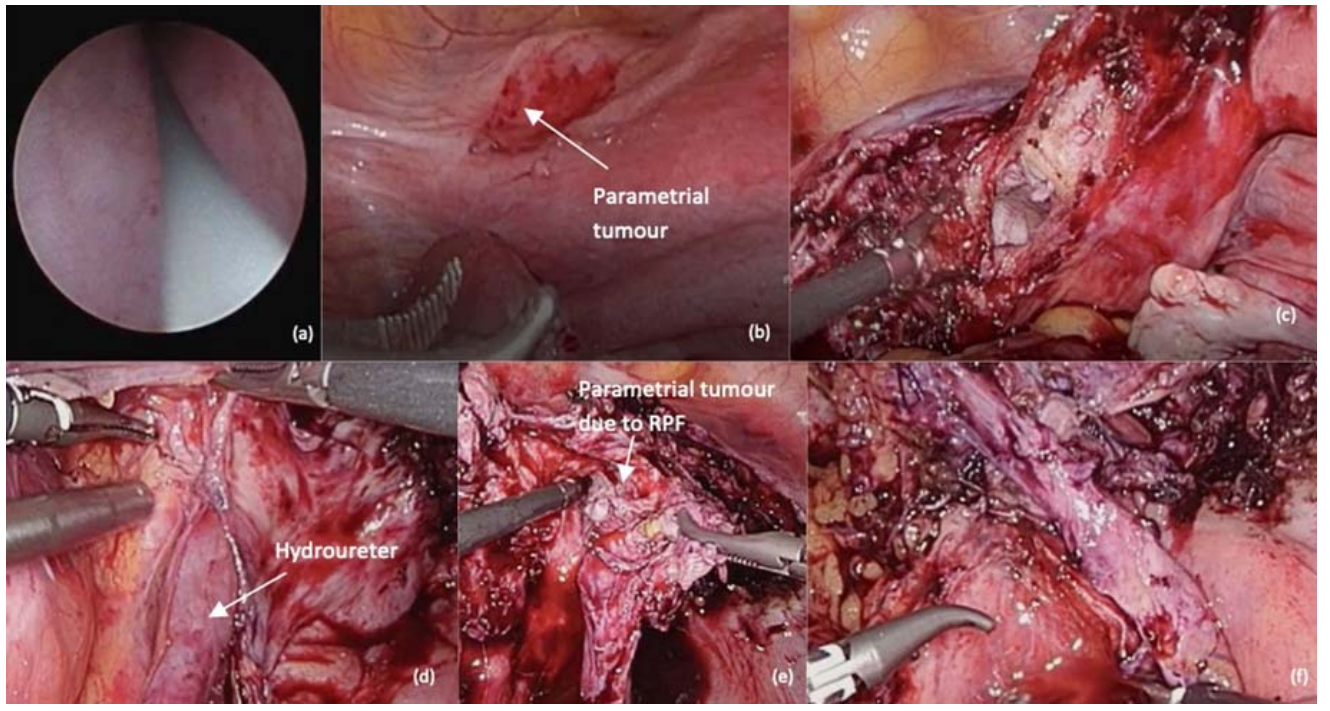

Figure 1.Laparoscopic view of resection of parametrial tumour due to IgG4 mediated retroperitoneal fibrosis

(a) Obstruction noted during cysto-ureteroscopy

(b) Appearance of parametrial tumour before resection

(c) Laparoscopic hysterectomy performed medial to parametrial tumour

(d) Hydroureter noted following ureterolysis

(e) Resection of parametrial tumour due to RPF around the ureter

(f) Relieving hydroureter and final outcome
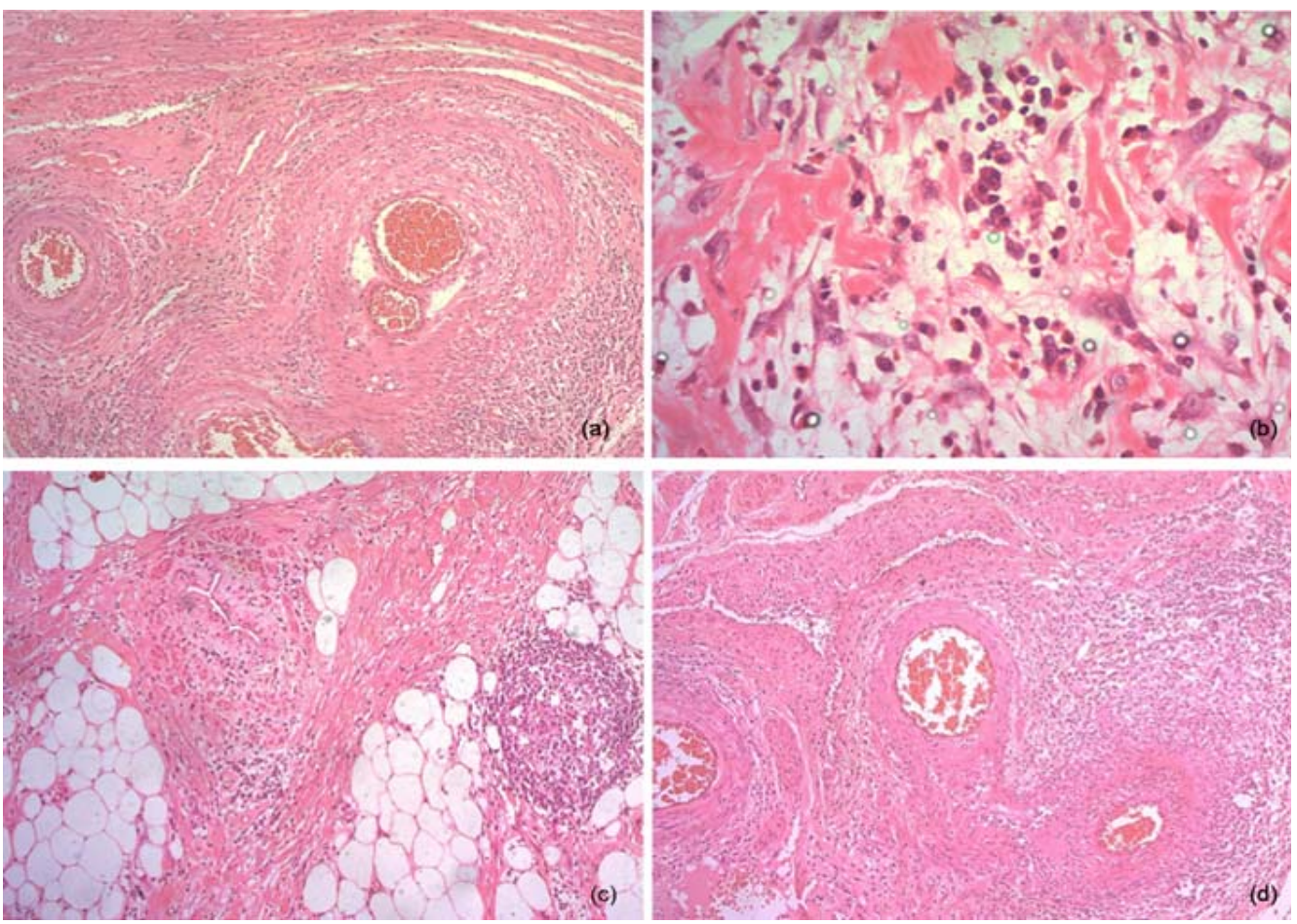

Figure 2. Histology of parametrial tumour due to IgG4 mediated retroperitoneal fibrosis

(a) $\mathrm{H} \& \mathrm{E} \times 100$. Onion skin-like fibrosis around vessels with narrowing of vessel lumina

(b) $\mathrm{H} \& \mathrm{E} \times 400$. Plasma cells and thick fibrous bundles

(c) $\mathrm{H} \& \mathrm{E} \times 100$. Fibrosis (arrowhead), lymphoid aggregate (arrow) and narrowing of vessel

(d) $\mathrm{H} \& \mathrm{E} \times 100$. Dense perivascular infiltrate with infiltration of vessel wall (lower right) by plasma cells and lymphocytes 


\section{Discussion}

This disorder is a rare clinical condition and has an incidence of 0.1 per 100,000 persons, and men are affected two to three-fold more often than women [5]. The mean age at presentation is 50-60 years. Presentation with urinary symptoms to Genito-Urinary surgeons is common in the disease entity concerned. However, because of its rarity among female patients, lack of standardized diagnostic criteria for IgG4-related retroperitoneal disease and the possibility of presenting as a parametrial tumour makes this disease a rare presentation in gynaecological practice.

Since it is often difficult to distinguish this from urothelial cancer, and rarely from cervical cancer clinically, histopathological evaluation of the retroperitoneal mass is necessary for diagnosis. CT-guided needle biopsy is sometimes useful in the diagnosis of retroperitoneal fibrosis [6]. However, in our patient, the cervix appeared healthy and her recent cervical smear was normal which warranted exclusion ureteric obstruction due to a possible ureteral endometriosis, which led to the laparoscopic excision of the tumour followed by tissue diagnosis.

Treating the patient on the basis of clinical diagnosis is an option described in Yoshino et al even in the background of a scenario mimicking a renal pelvic cancer [7]. However, authors believe that in severe symptomatic cases presenting with hydronephrosis and hydroureter and with the extended duration required for tumour regression following corticosteroids, surgical treatment is the superior option. It also gives an opportunity to exclude other differentials like pelvic endometriosis in female patients.

Gao W et al. presented 9 cases of ureteral obstruction due to external compression of the ureter that led to hydronephrosis and kidney dysfunction due to idiopathic retroperitoneal fibrosis managed with metallic ureteral stents. Placement of the metallic ureteral stents provided relief from ureteral obstruction and restored kidney function in all patients. However, some developed complications like stent migration, repeated metallic stent infections and haematuria [8]. This can be considered as a good option of management until the medical treatment takes effect. In our case, a cysto-ureteroscopy was performed prior to laparoscopy and an obstruction about 1-2 $\mathrm{cm}$ above the left cysto-ureteric junction was found, which resulted in a failure of stenting. Owing to this, and in order to exclude other differentials like pelvic endometriosis and pelvic tumours, a laparoscopic approach was decided upon.

Laparoscopy is a useful diagnostic tool and has the added advantage of being a modality of treatment at the same time. Commonly accepted benefits of the method, such as improved visualization of the pouch of Douglas, smaller incisions, decreased adhesion formation, reduced postoperative pain and shorter hospital stay favour a laparoscopic approach. However, other factors such as availability of suitable instruments and surgical expertise need to be considered before proceeding.

\section{Conclusion}

IgG4-related retroperitoneal fibrosis should be considered in the differential diagnosis of retroperitoneal and parametrial tumours amongst female patients, because it responds well to corticosteroid therapy and generally has a fair prognosis. The role of laparoscopy in treating this condition is plausible, considering it can be diagnostic and therapeutic. Despite its limiting factors such as the need for surgical expertise and suitable instruments, laparoscopic surgery for this condition supersedes medical therapy at the beginning, owing to faster symptom relief and the opportunity to exclude other pelvic pathology.

\section{Declarations}

Competing interests: The authors declare that they have no competing interests.

Patient consent: Informed consent was taken from the patient prior to writing.

Sources of funding: None of the authors received funding.

\section{Acknowledgements}

We would like to extend our gratitude to the theatre staff of operation theatre, King's Hospital, Colombo, Sri Lanka for their support in performing this complex surgery.

\section{References}

1. Tzou M, Gazeley DJ, Mason PJ. Retroperitoneal fibrosis. Vasc Med. 2014; 19(5): 407-14.

2. Chiba K, Kamisawa T, Tabata T, Hara S, Kuruma S, Fujiwara T, et al. Clinical features of 10 patients with IgG4-related retroperitoneal fibrosis. Intern Med. 2013; 52(14): 1545-51.

3. Lian L, Wang C, Tian JL. IgG4-related retroperitoneal fibrosis: a newly characterized disease. Int J Rheum Dis. 2016; 19(11): 1049-55.

4. Maritati F, Rocco R, Accorsi Buttini E, Marvisi C, Nicastro $\mathrm{M}$, Urban ML, et al. Clinical and prognostic significance of serum IgG4 in chronic periaortitis. An analysis of 113 patients. Front Immunol. 2019; 10: 693.

5. Uibu T, Oksa P, Auvinen A, Honkanen E, Metsärinne K, Saha $\mathrm{H}$, et al. Asbestos exposure as a risk factor for retroperitoneal fibrosis. Lancet 2004; 363(9419): 1422-26.

6. Aalberse RC, Stapel SO, Schuurman J, Rispens T. Immunoglobulin G4: an odd antibody. Clin Exp Allergy. 2009; 39(4): 469-77.

7. Yoshino T, Moriyama H, Fukushima M, Sanda N. A case of IgG4-related retroperitoneal fibrosis mimicking renal pelvic cancer. Urol Int. 2013; 90(3): 365-8.

8. Gao W, Ou T-W, Cui X, Wu J-T, Cui B. Metallic ureteral stent in restoring kidney function: Nine case reports. World J Clin Cases 2020; 8(13): 2841. 